\title{
Lauren classification combined with HER2 status is a better prognostic factor in Chinese gastric cancer patients
}

Miaozhen Qiu ${ }^{1,2 \dagger}$, Yixin Zhou ${ }^{1 \dagger}$, Xinke Zhang ${ }^{3 \dagger}$, Zixian Wang ${ }^{4}$, Fang Wang ${ }^{5}$, Jianyong Shao ${ }^{5}$, Jiabin Lu ${ }^{3}$, Ying Jin ${ }^{1}$, Xiaoli Wei ${ }^{1}$, Dongsheng Zhang ${ }^{1}$, Fenghua Wang ${ }^{1}$, Yuhong $\mathrm{Li}^{1}$, Dajun Yang ${ }^{6^{*}}$ and Ruihua $\mathrm{Xu}^{{ }^{*}}$

\begin{abstract}
Background: Lauren-classification and human epidermal growth factor receptor 2 (HER2) status are two important pathological features of gastric cancer patients. The prognostic value of HER2 in gastric cancer remains controversial. Intestinal type gastric cancer has better prognosis and higher HER2 positive proportion. What is the interaction between these two factors? We hypothesized that a combination of Lauren-classification and human epidermal growth factor receptor 2 (HER2) status (L-H status) might be more meaningful than either factor alone.

Methods: We collected 838 gastric cancer patients at all stages who had received treatment in our cancer center. This study was registered in the website of ClinicalTrials.Gov, with the number NCT01927146. We divided the patients into six groups according to their L-H status: Group A, HER2 negative and intestinal type; Group B, HER2 positive and intestinal type; Group C, HER2 negative and diffuse type; Group D, HER2 positive and diffuse type; Group E, HER2 negative and mixed type; and Group F, HER2 positive and mixed type.

Results: Diffuse type and intestinal type accounted for $51.0 \%$ and $33.9 \%$, respectively. The proportion of HER2 positive patients was $11.2 \%, 25.4 \%, 2.1 \%$ and $10.2 \%$ in the whole patient group, intestinal, diffuse and mixed type, respectively. Median overall survival was 34.0 months, 25.3 months, 27.6 months, 19.2 months, 25.9 months and 26.4 months in the six groups patients, $P=0.053$. There was a significant difference in survival among the first four groups $(P<0.001)$. HER2 was an independent prognostic factor in the intestinal type and in stage I+ II patients, but not in the diffuse type or stage III + IV patients. L-H status was an independent prognostic factor in patients at all stages. For the diffuse and intestinal types, the multivariate analysis showed that HER2 was not an independent prognostic factor, while Lauren classification and L-H status were. Moreover, L-H status was a better prognostic factor than the Lauren classification.
\end{abstract}

Conclusions: L-H status is a prognostic factor in diffuse and intestinal type patients, but not in the mixed type. Patients with HER2 negative and intestinal type had the best survival, while patients with HER2 positive status and diffuse type had the worst survival.

Keywords: Gastric cancer, Lauren classification, Human epidermal growth factor receptor 2, Prognosis

\footnotetext{
*Correspondence: yangdj@sysucc.org.cn; xurh@sysucc.org.cn

†Equal contributors

${ }^{6}$ Department of Experimental Research, Sun Yat-Sen University Cancer Center; State Key Laboratory of Oncology in South China; Collaborative Innovation Center for Cancer Medicine, 651 Dongfeng Road East, Guangzhou 510060, China 'Department of Medical Oncology, Sun Yat-Sen University Cancer Center; State Key Laboratory of Oncology in South China; Collaborative Innovation Center for Cancer Medicine, 651 Dongfeng Road East, Guangzhou 510060, China

Full list of author information is available at the end of the article
}

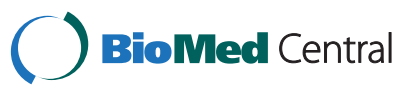

(c) 2014 Qiu et al.; licensee BioMed Central Ltd. This is an Open Access article distributed under the terms of the Creative Commons Attribution License (http://creativecommons.org/licenses/by/4.0), which permits unrestricted use, distribution, and reproduction in any medium, provided the original work is properly credited. The Creative Commons Public Domain Dedication waiver (http://creativecommons.org/publicdomain/zero/1.0/) applies to the data made available in this article, unless otherwise stated. 


\section{Background}

Gastric cancer is the second most common cause of cancer-related death worldwide [1]. The incidence of gastric carcinoma varies significantly from one part of the world to another and it is particularly common in Eastern Asia, especially in China [2]. Amplification, overexpression or both, of human epidermal growth factor receptor-2 (HER2, also known as ERBB2), a transmembrane receptor tyrosine kinase, is present in around $6.1-23.0 \%$ of gastric cancers [3-5]. In breast cancer, amplification and overexpression of the HER2 gene are associated with poor outcomes, higher mortality, higher recurrence and metastasis [6-8]. However, the prognostic value of HER2 status in gastric cancer remains controversial. Some studies showed that HER2-positive patients had a favorable survival [9-11], while other studies revealed no relationship between HER2 status and survival [4,12-14]. The majority of the publications showed that a HER2-postive status, measured by immunohistochemistry (IHC) or fluorescence in situ hybridization (FISH), was associated with poor survival and/ or clinicopathological characteristics, such as serosal invasion, lymph node metastases, disease stage, or distant metastases $[11,15,16]$.

Although the Lauren classification system dates back to 1965 , it is still widely accepted and employed by pathologists and physicians today. According to the Lauren classification, gastric adenocarcinomas can be divided into diffuse, intestinal and mixed type [17]. Cohesive cells that form gland-like structures characterize the intestinal type. For the diffuse type, tumor cells lack cell-to-cell interactions and infiltrate the stroma as single cells or small subgroups, leading to a population of non-cohesive, scattered tumor cells [17]. The intestinal-type is more frequent in males and in elderly patients, while the diffuse-type occurs more frequently in women and young patients [18]. Intestinal type patients have better outcomes than patients with diffuse-type tumors $[8,19-21]$. However, HER2 positivity is more common in intestinal-type gastric cancer [15]. The higher rate of HER2 positivity and better survival in the intestinal type is controversial. We hypothesized that the combination of the Lauren classification and HER2 status (L-H status) might be more helpful than either factor alone. In this study, we explored the relationship between Lauren classification and HER2 status; moreover, we also analyzed the prognostic value of L-H status.

\section{Methods}

\section{Patient collection}

From January 1996 to December 2006, we collected clinical information retrospectively from gastric cancer patients who received treatment in our cancer center. Patients included in the study met the following criteria: (1) histologically confirmed gastric adenocarcinoma patients that underwent gastrectomy; (2) adequate paraffin-embedded tumor tissue sample for pathological and HER2 status analysis; and (3) complete medical records with regular survival follow-up data. Overall survival (OS) data was present. The exclusion criteria were: (1) age <18 years old; and (2) other malignancy within the last 5 years, except carcinoma in situ of the cervix, or basal cell carcinoma.

All patients were categorized according to the $7^{\text {th }}$ American Joint Committee on Cancer (AJCC) TumorNode-Metastasis (TNM) stage.

\section{Lauren classification}

Assignment of histological type was based on the Lauren criteria. The intestinal type was described as a tumor with glandular architecture, resembling colonic carcinoma. The diffuse type was described as a tumor composed of solitary or small clusters of cells, and lacking glandular structures. The mixed type was described as the combination of these two features. Two pathologists reviewed the original diagnostic slides to make a diagnosis of Lauren classification.

\section{HER2 evaluation}

\section{Immunohistochemistry (IHC)}

For all patients, HER2 expression was detected by IHC. IHC staining was carried out using an anti-HER-2/NEU (4B5) antibody (Ventana Medical Systems, Inc. Tucson, AZ, USA) as the primary antibody against HER2 on a Ventana Benchmark XT automatic staining system, according to the manufacturer's instructions. The amended HER2 IHC scoring system for gastric cancer proposed by Hoffmann et al. was used as the criteria for scoring the stained slides [22].

\section{Fluorescence in situ hybridization (FISH)}

HER2 amplification levels were measured when the result of IHC was 2+. The PathVysion ${ }^{\oplus}$ HER2 DNA Probe kit (LSI ${ }^{\circ} H E R 2 /$ neu Spectrum Orange ${ }^{\mathrm{Tm}} /$ chromosome 7 centromere probe (CEP) ${ }^{\circledR} 17$ Spectrum Green) was used to perform FISH analysis, according to the manufacturer's protocol. A positive result from FISH was defined as a HER2:CEP17 ratio $\geq 2$.

Any case with IHC $3+$ or IHC2+/FISH + was considered to be HER2-positive, while cases with IHC 0 or IHC $1+$ or IHC 2+/FISH - were considered as HER2negative, according to criteria of the European Medicines Agency.

\section{L-H status}

We divided the patients into six groups according to their Lauren classification and HER2 status (L-H Status): Group A, HER2 negative and intestinal type; Group B, HER2 positive and intestinal type; Group C, HER2 negative and diffuse type; Group D, HER2 positive and diffuse type; Group E, HER2 negative and mixed type; and Group F, HER2 positive and mixed type. 


\section{Statistical analysis}

The Statistical Package of Social Sciences 13.0 software performed all the statistical analyses. A P value $<0.05$ was considered statistically significant. The Kaplan-Meier method was used to estimate OS. For patients who remained alive, data were censored at the date of the last contact. Kaplan-Meier analysis with log-rank testing was used for univariate analysis. OS was defined as the duration between the date of diagnosis and the date of last contact. Variables showing a trend for association with survival $(\mathrm{P}<0.05)$ and variables that were known to have prognostic value were selected for submission to a final multivariate Cox proportional hazards model, while variables that were highly associated with others were excluded from the final multivariate model. The chi-square test was used to compare the clinicopathological data.

We compared the -2log likelihood (which was the parameter in the Cox regression) of two different models of multivariate analysis: the smaller the value, the better the model [23].

\section{Ethics statement}

All patients signed written informed consent for their information to be used for the study. The independent ethics committees at the Cancer Center of Sun Yat-Sen University approved the study. The study was undertaken in accordance with the ethical standards of the World Medical Association Declaration of Helsinki.

This study was registered in the website of ClinicalTrials.Gov with a number of NCT01927146.

\section{Results}

\section{Patient demographics}

The median age of the 838 patients was 59 years (rang: 18 to 86 years); 554 were male and 284 were female. There were 88 stage IV patients at the time of diagnosis who all received gastrectomy to relieve the symptom of obstruction or bleeding. During follow-up, 91 patients developed distant metastasis and 12 patients had local recurrence. Until January 1, 2014, 77 patients had died from gastric cancer.

\section{Lauren classification}

There were $51.0 \%(427 / 838)$ of patients with diffuse type and $33.9 \%(284 / 838)$ patients with the intestinal type. The remaining 127 (15.1\%) patients belonged to the mixed type.

The relationship between clinicopathological features and Lauren classification is showed in Table 1. Among the patients who were younger than 60 years old, 269 (62.7\%) had the diffuse type, while for patients who were older than 59 years old; only 158 (38.6\%) patients had the diffuse type. The ratio of males to females was significantly higher in the intestinal-type than that in the diffuse-type (3.2 vs.
1.3; $\mathrm{P}<0.001)$. Patients in stages III and IV had a higher percentage of diffuse type than those in the stages I and II.

\section{HER2 status}

The percentages of IHC negative, $1+, 2+$ and $3+$ were $51.2 \%$ (429/838), 25.5\% (214/838), $15.4 \%$ (129/838) and $7.9 \%$ (66/838), respectively. For the IHC $2+$ patients, 28 patients were diagnosed as FISH positive. Thus, the proportion of patients positive for HER2 was 11.2\% (94/838) in the whole group of patients.

Among patients who were older than 60 years, there were more HER2 positive patents than among those younger than 59 years old. Stage IV patients had the highest proportion of HER2 positive (14.8\%). The relationship between clinicopathological features and HER2 status is shown in Table 1.

\section{L-H status}

The proportions of HER2 positive patients were $25.4 \%$, $2.1 \%$ and $10.2 \%$ in the intestinal type, diffuse type and mixed type, respectively $(\mathrm{P}<0.001)$. The median OS (from the time of diagnosis to the time of last contact) was 34.0 months, 25.3 months, 27.6 months, 19.2 months, 25.9 months and 26.4 months in the six groups of patients $(\mathrm{P}=0.053)$. Considering that the mixed type contained the features of diffuse type and intestinal type, the difference between diffuse and intestinal type could not be fully evaluated in the mixed type. In subsequent analyses we only evaluated the value of $\mathrm{L}-\mathrm{H}$ classification in the diffuse and intestinal types. The number of patients in these four groups was $212,72,418$ and nine respectively. The median survival was 34.0 months, 25.3 months, 27.6 months and 19.2 months $(\mathrm{P}<0.001$; Figure 1$)$. For the stage IV patients (including 88 concurrent metastasis and 91 metachronous metastasis patients), if we calculated the survival from the time of metastasis to the time of last contact, the median overall survival was 13.7 months, 10.2 months, 10.8 months and 7.9 months $(\mathrm{P}=0.001)$.

The relationship between L-H status and clinicopathological features is shown in Table 2. From the table, we could conclude that L-H status was a useful index. Among the four L-H groups, the clinicopathological features were quite different, except for the percentage of adjuvant chemotherapy.

\section{Survival analysis}

Both univariate and multivariable analyses were used to evaluate factors associated with OS. Factors of TNM stage $(\mathrm{P}<0.001)$, degree of differentiation $(\mathrm{P}=0.015)$, Lauren classification $(\mathrm{P}=0.006)$, HER2 status $(\mathrm{P}=0.033)$ and $\mathrm{L}-\mathrm{H}$ status $(P=0.003)$ were all significantly associated with $O S$ in the univariate analysis.

To further explore the prognostic value of HER2, we analyzed the survival difference between HER2 positive 
Table 1 Baseline characteristics

\begin{tabular}{|c|c|c|c|c|c|c|c|}
\hline & \multicolumn{3}{|c|}{ Lauren classification } & \multirow[t]{2}{*}{$P \#$ value } & \multicolumn{2}{|l|}{ HER2 status } & \multirow[t]{2}{*}{$P *$ value } \\
\hline & Diffuse(\%) & Intestinal(\%) & Mixed(\%) & & Negative(\%) & Positive(\%) & \\
\hline Sex & & & & $<0.0$ & & & \\
\hline Male & $242(43.7)$ & $216(39.0)$ & $96(17.3)$ & 01 & $486(87.7)$ & $68(12.3)$ & 0.176 \\
\hline Female & $185(64.7)$ & $68(23.9)$ & $31(10.9)$ & & $258(90.8)$ & $26(9.2)$ & \\
\hline Age & & & & $<0.001$ & & & $<0.001$ \\
\hline$\leq 59$ & $269(62.7)$ & $104(24.2)$ & $56(13.1)$ & & $398(92.8)$ & $31(7.2)$ & \\
\hline$>59$ & 158 (38.6) & $180(44.0)$ & $71(17.4)$ & & $346(84.6)$ & $63(15.4)$ & \\
\hline Stage & & & & $<0.001$ & & & 0.406 \\
\hline 1 & $68(47.9)$ & $60(42.3)$ & $14(9.8)$ & & $131(92.3)$ & $11(7.7)$ & \\
\hline$\|$ & $96(41.7)$ & $102(44.3)$ & $32(13.9)$ & & $203(88.3)$ & $27(11.7)$ & \\
\hline III & $215(56.9)$ & $95(25.1)$ & $68(18.0)$ & & 335 (88.6) & $43(11.4)$ & \\
\hline IV & $48(54.5)$ & $27(30.7)$ & $13(14.8)$ & & $75(85.2)$ & $13(14.8)$ & \\
\hline Degree of differentiation & & & & $<0.001$ & & & $<0.001$ \\
\hline Well + Moderate & $0(0)$ & $262(76.2)$ & $82(23.8)$ & & $270(78.5)$ & $74(21.5)$ & \\
\hline Poor + signet ring cell & $427(86.4)$ & $22(4.5)$ & $45(9.1)$ & & $474(96.0)$ & $20(4.0)$ & \\
\hline Location & & & & $<0.001$ & & & $<0.001$ \\
\hline Proximal & $110(35.9)$ & $146(47.7)$ & $50(16.3)$ & & $251(82.0)$ & $55(18.0)$ & \\
\hline Distal & $276(59.1)$ & $128(27.4)$ & $63(13.5)$ & & $436(93.4)$ & $31(6.6)$ & \\
\hline Total stomach & $41(63.1)$ & $10(15.4)$ & $14(21.5)$ & & $57(87.7)$ & $8(12.3)$ & \\
\hline \multicolumn{8}{|l|}{ Adjuvant chemotherapy } \\
\hline Yes & $302(51.4)$ & $192(32.7)$ & $94(15.9)$ & & $531(90.3)$ & $57(9.7)$ & \\
\hline No & 77 (47.5) & 65 (40.1) & 20 (12.4) & 0.170 & 138 (85.2) & 24 (14.8) & 0.063 \\
\hline
\end{tabular}

HER2: human epidermal growth factor receptor 2.

$\# P$ values of Lauren classification in different clinical features. ${ }^{*} P$ values of HER 2 status in different clinical features.

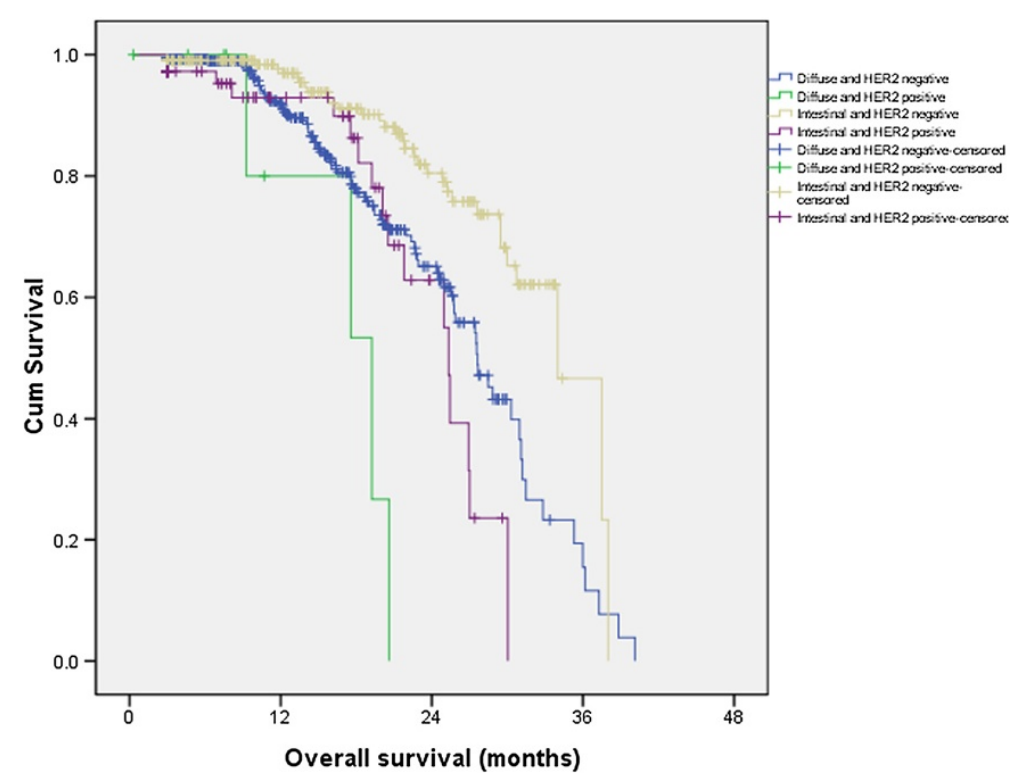

Figure 1 The survival difference among different L-H status. 
Table 2 The relationship between different L-H status and clinical features

\begin{tabular}{|c|c|c|c|c|c|}
\hline & Group A & Group B & Group C & Group D & $P$ value \\
\hline \multicolumn{6}{|l|}{ Sex } \\
\hline Male & 163 & 54 & 235 & 6 & \\
\hline Female & 49 & 18 & 183 & 3 & $<0.001$ \\
\hline \multicolumn{6}{|l|}{ Age } \\
\hline$\leq 59$ & 82 & 21 & 264 & 6 & \\
\hline$>59$ & 130 & 51 & 154 & 3 & $<0.001$ \\
\hline \multicolumn{6}{|l|}{ Stage } \\
\hline । & 48 & 10 & 69 & 1 & \\
\hline$\|$ & 80 & 23 & 93 & 2 & \\
\hline III & 66 & 30 & 211 & 3 & \\
\hline IV & 18 & 9 & 45 & 3 & $<0.001$ \\
\hline \multicolumn{6}{|l|}{$\begin{array}{l}\text { Degree of } \\
\text { differentiation }\end{array}$} \\
\hline Well + Moderate & 193 & 69 & 0 & 0 & \\
\hline Poor + signet ring cell & 19 & 3 & 418 & 9 & $<0.001$ \\
\hline \multicolumn{6}{|l|}{ Location } \\
\hline Proximal & 99 & 45 & 111 & 1 & \\
\hline Distal & 104 & 24 & 269 & 7 & \\
\hline Total stomach & 9 & 3 & 38 & 1 & $<0.001$ \\
\hline \multicolumn{6}{|l|}{$\begin{array}{l}\text { Adjuvant } \\
\text { chemotherapy }\end{array}$} \\
\hline Yes & 144 & 48 & 297 & 5 & \\
\hline No & 50 & 15 & 76 & 1 & 0.505 \\
\hline
\end{tabular}

Group A, HER2 negative and intestinal type; Group B, HER2 positive and intestinal type; Group C, HER2 negative and diffuse type; Group D, HER2 positive and diffuse type.

and HER2 negative patients in intestinal type and diffuse type, respectively. We found that HER2 positivity was an independent adverse prognostic factor in the intestinal type $(P<0.001)$, but not in the diffuse type $(P=0.084$; Figure 2A, B).

We then analyzed the prognostic value of HER2 positivity in different stages. HER2 positivity was an independent adverse prognostic factor in stage I and II patients $(\mathrm{P}<0.001)$, but not in stage III and IV patients $(\mathrm{P}=0.125$; Figure $2 \mathrm{C}, \mathrm{D})$.

For the multivariable regression analysis, we first set up a model (model A) that included age, gender, degree of differentiation, TNM stage, Lauren classification and HER2 status. Model A showed that age, degree of differentiation, TNM stage and Lauren classification were independent factors for OS $(P=0.001,0.017,<0.001$ and 0.047 , respectively, Table 3$)$. HER2 status was not an independent prognostic factor $(\mathrm{P}=0.285)$. The $-2 \log$ likelihood was 1663.155. We then set up another model (model B), which was identical to the first one except that the Lauren classification and HER2 status were replaced by the L-H status. In model B, TNM stage and $\mathrm{L}-\mathrm{H}$ status were independent factors for OS $(\mathrm{P}=0.028$, $<0.001$ and 0.006 , respectively, Table 3 ). The $-2 \log$ likelihood was 1411.610.

We also analyzed the prognostic value of $\mathrm{L}-\mathrm{H}$ status in different stages. L-H status was an independent prognostic factor in both early stage (I and II) patients $(\mathrm{P}<$ $0.001)$ and advanced stage (III and IV) patients $(\mathrm{P}=0.036$; Figure 3A, B).

\section{Discussion}

The prognostic value of HER2 status in gastric cancer remains controversial. Some studies reported that HER2 positivity was an adverse prognostic factor, while some found that it indicated better survival. Other studies even considered that it had no relationship with survival. Based on the Lauren classification, gastric cancers could be divided into the diffuse type, intestinal type and mixed type. The intestinal type has a better survival than the diffuse type. However, intestinal type patients were more likely to be HER2 than diffuse type patients. In our study, HER2 was not an independent prognostic factor for gastric cancer patients in the multivariate analysis. When we separated the patients into diffuse and intestinal types, we found that HER2 was an independent adverse prognostic factor for the intestinal type. We also analyzed the prognostic value of HER2 positivity in patients at different stages. HER2 positivity was an independent prognostic factor for stage I and II patients, but not in stage III and IV patients. This was different from the result of Kataoka et al. [14]. They analyzed 213 Japanese gastric cancer patients retrospectively and found that the OS of HER2-negative and -positive patients was not significantly different in the whole group patients. However, in patients with stage III/IV, they found that the OS was worse in HER2-positive patients $(\mathrm{P}=0.0149)$ [14]. In the 2012 European Society for Medical Oncology (ESMO) conference, a multicenter study conducted by Kurokawa et al. showed that HER 2 positivity was an independent prognostic factor in stage I and II patients, but not in stage III and IV patients [24]. This was consistent with our results. These were all retrospective analyses. Therefore, prospective studies are required to explore the prognostic value of HER2 in early stage gastric cancer patients.

Based on the analysis above, we hypothesized that when we discussed the prognostic value of HER2 positive, there were other factors that should be into consideration, such as the TNM stage and the Lauren classification.

HER2 positivity was much more common in the proximal, intestinal type and stage IV gastric cancer patients. Male, older patients and proximal gastric cancer patients had a higher percentage of intestinal type. These basic 


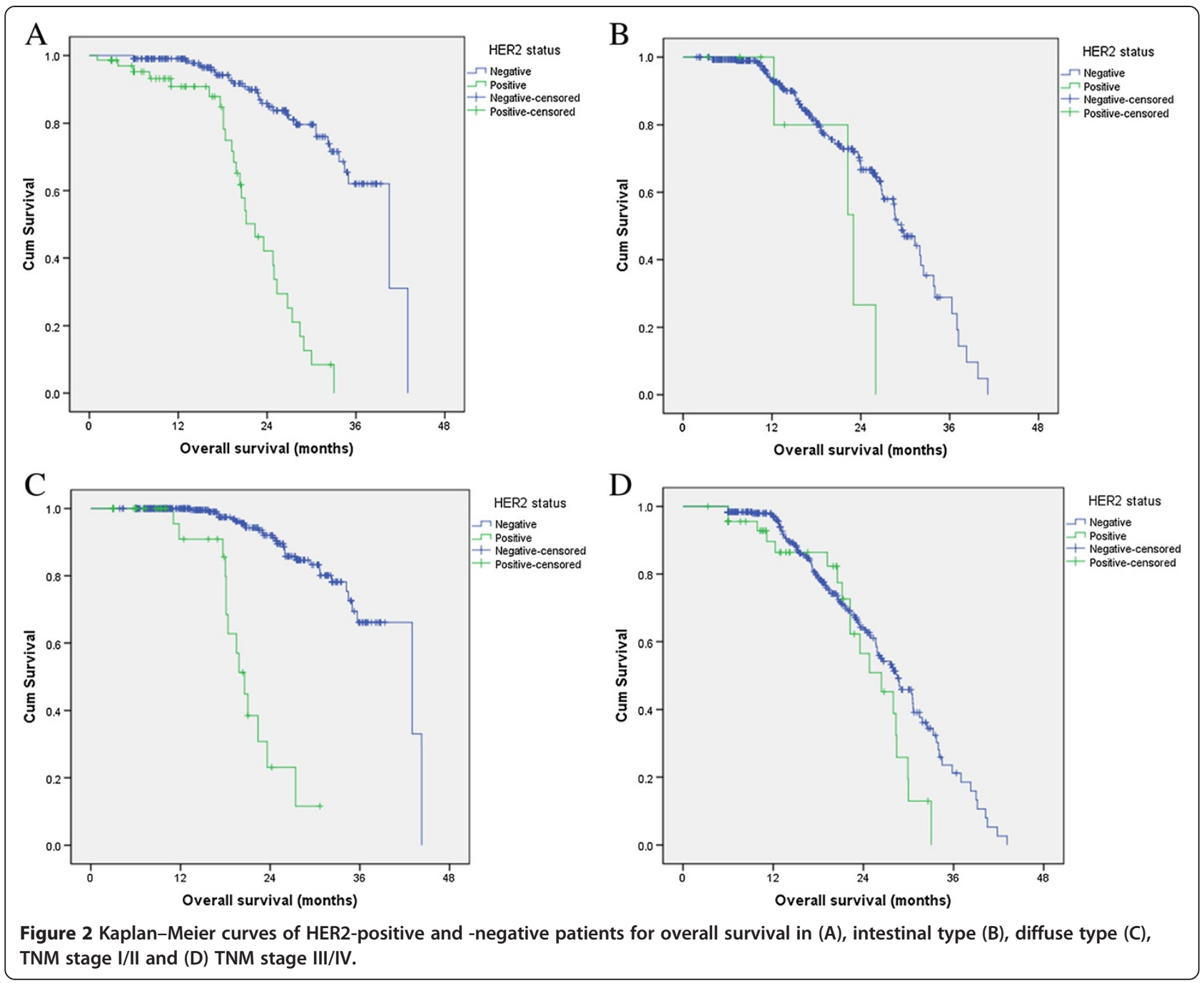

clinicopathological features were all consistent with previous studies [4,15,16,18-21].

In the 2014, at the American Society of Clinical Oncology (ASCO) annual meeting, both HER2 positivity and the Lauren classification were considered as the most important progresses in gastric cancer in the last 50 years. These were two important pathological features of gastric cancer. In the present study, we combined these two factors together and proposed the concept of L-H status. Since mixed type was not a pure group, L-H status is not a good option for mixed

Table 3 The multivariable analysis of overall survival in gastric carcinoma

\begin{tabular}{|c|c|c|c|c|c|c|}
\hline & Model A & & & Model B & & \\
\hline & Hazard ratio & $95 \% \mathrm{Cl}$ & $P$ value & Hazard ratio & $95 \% \mathrm{Cl}$ & $P$ value \\
\hline Gender & 1.208 & $0.736-1.983$ & 0.455 & 0.984 & $0.566-1.711$ & 0.954 \\
\hline Age & 2.302 & $1.383-3.831$ & 0.001 & 1.472 & $1.059-2.047$ & 0.028 \\
\hline Stage & 3.604 & $2.551-5.091$ & $<0.001$ & 3.610 & $2.490-5.233$ & $<0.001$ \\
\hline Degree of differentiation & 0.505 & $0.288-0.886$ & 0.017 & 0.424 & $0.167-1.074$ & 0.070 \\
\hline Lauren classfication & 1.440 & $1.004-2.066$ & 0.047 & - & - & - \\
\hline HER2 status & 0.669 & $0.320-1.398$ & 0.285 & - & - & - \\
\hline L-H status & - & - & - & 2.222 & $1.259-3.920$ & 0.006 \\
\hline
\end{tabular}

Abbreviations: $\mathrm{Cl}$ confidence interval, HER2 human epidermal growth factor receptor-2, $\mathrm{L}-\mathrm{H}$ status Lauren classification and HER2 status. Model A includes the factors of Lauren classification and HER2 status; Model B includes the combination factor of L-H status. 

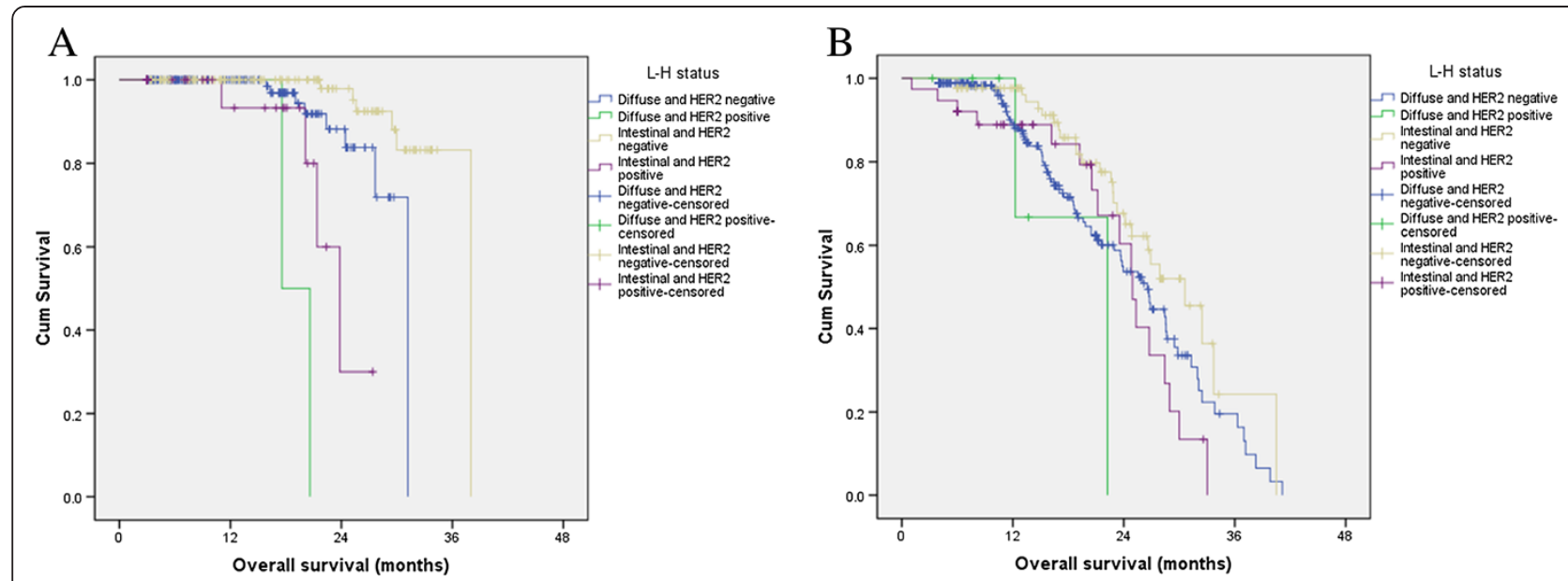

Figure 3 Kaplan-Meier curves of L-H status for overall survival in (A), TNM stage I/II and (B) TNM stage III/IV.

type. We only considered intestinal and diffuse type in the analysis. We divided the gastric cancer patients according to their L-H status to create four groups: Group A, HER2 negative and intestinal type; Group B, HER2 positive and intestinal type; Group C, HER2 negative and diffuse type; and Group D, HER2 positive and diffuse type. Group C had the largest number of patients. Unsurprisingly, the patients in Group A had the best prognosis, while those in Group D had the worst. Although both intestinal type and L-H status were independent prognostic factors in the multivariate analysis, the -2log likelihood was smaller in the L-H status model: the smaller the value of this statistic, the better the model. Therefore, the $\mathrm{L}-\mathrm{H}$ status was better than the Lauren classification for predicting the prognosis.

In the multivariate analysis, age, TNM stage and L-H status were all independent prognostic factors for gastric adenocarcinoma patients. The L-H status could replenish the TNM stage. Moreover, we found that L-H status was an independent prognostic factor in stage I + II and stage III + IV patients. Although the L-H status was not useful in the mixed type, we recommend that all the gastric cancer patients should be subjected to Lauren classification and their HER2 status checked to determine their L-H status. It is not only helpful to evaluate prognosis, but also is helpful to decide treatment. For HER2 positive metastasis gastric cancer patients, trastuzumab is the standard treatment.

The limitations of the present study are: 1) its retrospective nature from a single-institution; 2) the fact that the impact of various treatment-related outcomes could not be fully evaluated; and 3) that progression free survival or disease free survival could not be fully analyzed.

External validation using other large databases or prospective studies to evaluate the prognostic effect of L-H status is required. The underlying mechanism of intestinal type gastric cancer and relationship with high HER2 expression requires further exploration.

\section{Conclusions}

In this large sample size study, we found that HER2 positivity was not an independent prognostic factor in the whole group of patients, but it was in the intestinal type and stage I and II patients. The combination of the Lauren classification and HER2 status (L-H status) was a better prognostic factor than the Lauren classification alone in the diffuse and intestinal type. We recommend that all the gastric cancer patients should be subjected to Lauren classification and their HER2 status checked to determine their L-H status.

\section{Abbreviations}

HER2: Human epidermal growth factor receptor-2;

IHC: Immunohistochemistry; FISH: Fluorescence in situ hybridization; L-H status: Lauren classification and HER2 status; OS: Overall survival;

AJCC: American Joint Committee on Cancer; TNM: Tumor-Node-Metastasis;

CEP: Centromere probe; ESMO: European Society for Medical Oncology;

ASCO: American Society of Clinical Oncology.

\section{Competing interests}

We have no financial or personal relationships with other people or organizations that would bias our work.

This work was supported by:

1. The third outstanding young talents training plan of Sun Yat-sen University cancer center (No. 04140501\#)

2. Medical Scientific Research of Guangdong province B2014161.

3. National High Technology Research and Development Program of China (863 Program), China (No. 2012AA02A506).

4. Natural Science Foundation of China (No. 81372570).

5. The Science and Technology Department of Guangdong Province, China (No. 2012B031800088).

6. This study is supported by Shanghai Roche Pharmaceuticals Ltd (ML28674).

\section{Authors' contributions}

QMZ and ZYX participated in the clinical data collecting and drafted the manuscript. ZXK and LJB carried out the $\mathrm{HC}$ and Lauren classification analysis. WF and SJY carried out the FISH. JY, WZX and WXL participated in the clinical data collecting of the gastric carcinoma patients. ZDS and WFH performed the statistical analysis. LYH participated in the design of the study. YDJ and XRH conceived of the study, and participated in its design and coordination and helped to draft the manuscript. All authors read and approved the final manuscript. 


\section{Acknowledgements}

We gratefully thank the staff members in the Department of Medical Oncology and GI Surgery Oncology at Sun Yat-sen University Cancer Center for their suggestion and assistance.

\section{Author details}

${ }^{1}$ Department of Medical Oncology, Sun Yat-Sen University Cancer Center; State Key Laboratory of Oncology in South China; Collaborative Innovation Center for Cancer Medicine, 651 Dongfeng Road East, Guangzhou 510060, China. ${ }^{2}$ The Sidney Kimmel Comprehensive Cancer Center, The Johns Hopkins University School of Medicine, Baltimore, MD 21231, USA. ${ }^{3}$ Department of pathology, Sun Yat-Sen University Cancer Center; State Key Laboratory of Oncology in South China; Collaborative Innovation Center for Cancer Medicine, 651 Dongfeng Road East, Guangzhou 510060, China. ${ }^{4}$ Faculty of medical sciences, Sun Yat-sen University, Guangzhou Zhongshaner Road No. 74, Guangzhou, China. ${ }^{5}$ Department of Molecular Pathology, Sun Yat-Sen University Cancer Center; State Key Laboratory of Oncology in South China; Collaborative Innovation Center for Cancer Medicine, 651 Dongfeng Road East, Guangzhou, China. ${ }^{6}$ Department of Experimental Research, Sun Yat-Sen University Cancer Center; State Key Laboratory of Oncology in South China; Collaborative Innovation Center for Cancer Medicine, 651 Dongfeng Road East, Guangzhou 510060, China.

Received: 24 July 2014 Accepted: 23 October 2014 Published: 7 November 2014

\section{References}

1. Kamangar F, Dores GM, Anderson WF: Patterns of cancer incidence, mortality, and prevalence across five continents: defining priorities to reduce cancer disparities in different geographic regions of the world. J Clin Oncol 2006, 24(14):2137-2150.

2. Moore MA, Eser S, Igisinov N, Igisinov S, Mohagheghi MA, Mousavi-Jarrahi A, Ozenturk G, Soipova M, Tuncer M, Sobue T: Cancer epidemiology and control in North-Western and Central Asia - past, present and future. Asian Pac J Cancer Prev 2010, 11(Suppl 2):17-32.

3. Hsu JT, Chen TC, Tseng JH, Chiu CT, Liu KH, Yeh CN, Hwang TL, Jan YY, Yeh TS: Impact of HER-2 overexpression/amplification on the prognosis of gastric cancer patients undergoing resection: a single-center study of 1,036 patients. Oncologist 2011, 16(12):1706-1713.

4. Sheng WQ, Huang D, Ying JM, Lu N, Wu HM, Liu YH, Liu JP, Bu H, Zhou XY, DU X: HER2 status in gastric cancers: a retrospective analysis from four Chinese representative clinical centers and assessment of its prognostic significance. Ann Oncol 2013, 24(9):2360-2364.

5. Bang YJ, Van Cutsem E, Feyereislova A, Chung HC, Shen L, Sawaki A, Lordick F, Ohtsu A, Omuro Y, Satoh T, Aprile G, Kulikov E, Hill J, Lehle M, Rüschoff J, Kang YK, ToGA Trial Investigators: Trastuzumab in combination with chemotherapy versus chemotherapy alone for treatment of HER2-positive advanced gastric or gastro-oesophageal junction cancer (ToGA): a phase 3, open-label, randomised controlled trial. Lancet 2010, 376(9742):687-697.

6. Seshadri R, Horsfall DJ, Firgaira F, McCaul K, Setlur V, Chalmers AH, Yeo R, Ingram D, Dawkins $H$, Hahnel R: The relative prognostic significance of total cathepsin D and HER-2/neu oncogene amplification in breast cancer. The South Australian Breast Cancer Study Group. Int I Cancer 1994, 56(1):61-65.

7. Slamon DJ, Clark GM, Wong SG, Levin WJ, Ullrich A, McGuire WL: Human breast cancer: correlation of relapse and survival with amplification of the HER-2/neu oncogene. Science 1987, 235(4785):177-182.

8. Toikkanen S, Helin H, Isola J, Joensuu H: Prognostic significance of HER-2 oncoprotein expression in breast cancer: a 30-year follow-up. J Clin Oncol 1992, 10(7):1044-1048.

9. Gomez-Martin C, Garralda E, Echarri MJ, Ballesteros A, Arcediano A Rodriguez-Peralto JL, Hidalgo M, Lopez-Rios F: HER2/neu testing for antiHER2-based therapies in patients with unresectable and/or metastatic gastric cancer. J Clin Pathol 2012, 65(8):751-757.

10. Jain S, Filipe MI, Gullick WJ, Linehan J, Morris RW: c-erbB-2 proto-oncogene expression and its relationship to survival in gastric carcinoma: an immunohistochemical study on archival material. Int J Cancer 1991, 48(5):668-671.

11. Jorgensen JT, Hersom M: HER2 as a prognostic marker in gastric cancer a systematic analysis of data from the literature. J Cancer 2012, 3:137-144.
12. He C, Bian XY, Ni XZ, Shen DP, Shen YY, Liu H, Shen ZY, Liu Q: Correlation of human epidermal growth factor receptor 2 expression with clinicopathological characteristics and prognosis in gastric cancer. World J Gastroenterol 2013, 19(14):2171-2178.

13. Janjigian $Y Y$, Werner D, Pauligk C, Steinmetz K, Kelsen DP, Jager E, Altmannsberger HM, Robinson E, Tafe LJ, Tang LH, Shah MA, Al-Batran SE: Prognosis of metastatic gastric and gastroesophageal junction cancer by HER2 status: a European and USA International collaborative analysis. Ann Oncol 2012, 23(10):2656-2662.

14. Kataoka Y, Okabe H, Yoshizawa A, Minamiguchi S, Yoshimura K, Haga H, Sakai Y: HER2 expression and its clinicopathological features in resectable gastric cancer. Gastric Cancer 2013, 16(1):84-93.

15. Qiu MZ, Li Q, Wang ZQ, Liu TS, Liu Q, Wei XL, Jin Y, Wang DS, Ren C, Bai L, Wei XL, Jin Y, Wang DS, Ren C, Bai L, Zhang DS, Wang FH, Li YH, Xu RH: HER2-positive patients receiving trastuzumab treatment have a comparable prognosis with HER2-negative advanced gastric cancer patients: a prospective cohort observation. Int J Cancer 2014, 134(10):2468-2477.

16. Liang JW, Zhang JJ, Zhang T, Zheng ZC: Clinicopathological and prognostic significance of HER2 overexpression in gastric cancer: a meta-analysis of the literature. Tumour Biol 2014, 35(5):4849-4858.

17. Lauren P: THE two histological main types of gastric carcinoma: diffuse and so-called intestinal-type carcinoma. an attempt at a histo-clinical classification. Acta Pathol Microbiol Scand 1965, 64:31-49.

18. Qiu MZ, Cai MY, Zhang DS, Wang ZQ, Wang DS, Li YH, Xu RH: Clinicopathological characteristics and prognostic analysis of Lauren classification in gastric adenocarcinoma in China. J Transl Med 2013, 11:58.

19. Tanner M, Hollmen M, Junttila TT, Kapanen Al, Tommola S, Soini Y, Helin $H$ Salo J, Joensuu H, Sihvo E, Elenius K, Isola J: Amplification of HER-2 in gastric carcinoma: association with Topoisomerase llalpha gene amplification, intestinal type, poor prognosis and sensitivity to trastuzumab. Ann Oncol 2005, 16(2):273-278.

20. Yamashita K, Sakuramoto S, Katada N, Futawatari N, Moriya H, Hirai K, Kikuchi S, Watanabe M: Diffuse type advanced gastric cancer showing dismal prognosis is characterized by deeper invasion and emerging peritoneal cancer cell: the latest comparative study to intestinal advanced gastric cancer. Hepato-Gastroenterology 2009, 56(89):276-281.

21. Zheng H, Takahashi H, Murai Y, Cui Z, Nomoto K, Miwa S, Tsuneyama K, Takano Y: Pathobiological characteristics of intestinal and diffuse-type gastric carcinoma in Japan: an immunostaining study on the tissue microarray. J Clin Pathol 2007, 60(3):273-277.

22. Gianni L, Eiermann W, Semiglazov V, Manikhas A, Lluch A, Tjulandin S, Zambetti M, Vazquez F, Byakhow M, Lichinitser M, Climent MA, Ciruelos E, Ojeda B, Mansutti M, Bozhok A, Baronio R, Feyereislova A, Barton C, Valagussa P, Baselga J: Neoadjuvant chemotherapy with trastuzumab followed by adjuvant trastuzumab versus neoadjuvant chemotherapy alone, in patients with HER2-positive locally advanced breast cancer (the NOAH trial): a randomised controlled superiority trial with a parallel HER2-negative cohort. Lancet 2010, 375(9712):377-384.

23. Harrell FE Jr, Califf RM, Pryor DB, Lee KL, Rosati RA: Evaluating the yield of medical tests. JAMA 1982, 247(18):2543-2546.

24. Kurokawa Y, Matsuura N, Kimura Y, Kawabata R, Adachi S, Fujita J, Nishikawa K, Takiguchi S, Mori M, Doki Osaka/JP Y: Survival impact of HER2 status in patients with gastric cancer: a multicenter large-scale study in Japan. ESMO Abstr 2012, 677P. http://abstracts.webges.com/myitinerary/session-296.html? congress=esmo 2012 .

doi:10.1186/1471-2407-14-823

Cite this article as: Qiu et al: Lauren classification combined with HER2 status is a better prognostic factor in Chinese gastric cancer patients. BMC Cancer 2014 14:823. 